\title{
ESTABILIDADE TÉRMICA E FLAMABILIDADE DE NANOCOMPÓSITOS DE POLIETILENO DE ALTA MASSA MOLAR PRODUZIDOS POR POLIMERIZAÇÃO IN SITU
}

\author{
Sara Pereira de Agrela ${ }^{\prime}$ \\ Luiz Rogério Pinho de Andrade Lima' \\ Rosemário Cerqueira Souza '
}

\section{Resumo}

Nanocompósitos de polietileno de alta massa molar com argila, grafita ou talco foram produzidos utilizando polimerização in situ direta em solvente usando um sistema catalítico tipo Ziegler $\left(\mathrm{TiCl}_{4}\right.$ e trietilalumínio em hexano). O polímero produzido apresentou uma massa molar alta e degradação térmica em atmosfera inerte em temperatura acima de $400^{\circ} \mathrm{C}$. A degradação térmica dos polímeros nos nanocompósitos foi deslocada para temperaturas um pouco mais altas (até próximo de $500^{\circ} \mathrm{C}$ ) indicando um aumento da estabilidade térmica da matriz polimérica. Os testes de flamabilidade mostraram um efeito de redução ou atenuação da progressão das chamas para todos os nano-compósitos devido ao efeito de barreira para gases e compostos voláteis.

Palavras-chave: Polietileno; Argila; Grafita; Talco; Nanocompósito; Polimerização in situ; Flamabilidade.

\section{THERMAL STABILITY AND INFLAMMABILITY OF NANOCOMPOSITES OF HIGH DENSITY POLYETHYLENE OBTAINED BY IN SITU POLYMERIZATION}

\begin{abstract}
High density polyethylene-clay, graphite or talc composites were produced using direct solvent in situ polymerization using a Ziegler catalyst system ( $\mathrm{TiCl}_{4}$ and triethylaluminum in hexane). The produced polymer had a high average molecular weight and in inert atmosphere presents a thermal degradation above $400^{\circ} \mathrm{C}$. The thermal degradation of the polymers in the nanocomposites was shifted for higher temperature (until close to $500^{\circ} \mathrm{C}$ ) indicating an improvement in the thermal stability of the polymeric matrix. The inflammability tests clearly showed a retardation or attenuation effect of the flames progression for all nanocomposites due to the barrier effect for gases and volatile compounds.
\end{abstract}

Keywords: Polyethylene; Clay; Graphite; Talc; Nanocomposite; In situ polymerization; Flammability.

\section{INTRODUÇÃO}

Nanocompósitos tem recebido considerável atenção nos últimos anos devido à melhoria nas propriedades mecânicas e devido ao aumento da estabilidade térmica, resistência ao fogo e temperatura de ignição [I-3]. Nanocompósitos de poliolefinas são materiais muito promissores, mas o caráter apolar destes polímeros dificulta a produção deste material, especialmente usando o método de extrusão. Para matrizes poliolefínicas o método de polimerização in situ tem se mostrado mais efetivo sobretudo para cargas compostas por argila ou materiais planares [I]. Neste trabalho é enfocada a produção e a caracterização da estabilidade térmica e flamabilidade de nanocompósitos de polietileno de alta massa molar produzidos usando o sistema catalítico convencional de Ziegler-Natta.

Vários estudos focam a estabilidade térmica e a redução da flamabilidade de polímeros em nanocompósitos produzidos por extrusão, entretanto são escassos os estudos sobre a estabilidade térmica de polietileno de alta massa molar em nanocompósitos produzidos por polimerização in situ [ $1,4,5]$. Jin et al. [6] produziu nanocompósitos de polietileno por polimerização in situ usando montmorilonita sódica e montmorilonita organofílica intercalada com $\mathrm{TiCl}_{4}$ e ativada com trietil alumínio (TEAL). Os resultados indicaram a obtenção de um polímero com alta massa

'Departamento de Ciência e Tecnologia dos Materiais, Escola Politécnica, Universidade Federal da Bahia, Salvador, BA, Brasil. E-mail: lelo@ufba.br 
molar e cristalinidade em torno de $35 \%$. As camadas da argila ficaram bem disperses na matriz polimérica. Ramazani et al. [7] produziu nanocompósitos de polietileno e montemorillonita modificada por polimerização in situ. A argila foi ativada usando triisobutil alumínio (TIBA) ou TEAL a posteriormente $\mathrm{TiCl}_{4}$ e etileno foram usados na polimerização. Foi usado de 2 a $9 \%$ de argila e o polímero produzido apresentou temperatura de fusão entre 132 e $133^{\circ} \mathrm{C}$, cristalinidade de 66 a $70 \%$ e estabilidade térmica ate $400{ }^{\circ} \mathrm{C}$. Nikkhah et al. [8] investigaram as propriedades de nanocompósitos de polietileno e argila usando polimerização in situ e um catalisador bi-suportado (bentonita e $\mathrm{MgCl}_{2} / \mathrm{TiCl}_{4}$ ). Os resultados indicaram que as temperaturas de cristalização nos nanocompósitos foram mais altas que o polietileno puro e que houve uma melhoria na estabilidade térmica do polímero, causada pela boa dispersão e esfoliação da argila. Abedi et al. [9] produziram nanocompósitos de polietileno e argila por polimerização in situ usando $\mathrm{TiCl}_{4}, \mathrm{TEAL}$ e um organo-metálico modificado butil octil magnésio (BOM) como catalisador. As camadas da argila foram esfoliadas o que melhorou a estabilidade térmica dos nanocompósitos em relação ao polietileno puro.

O objetivo deste estudo é a produção de nanocompósitos de polietileno de alta massa molar e carga mineral composta por argila, grafita ou talco usando polimerização in situ diretamente no solvente com um sistema catalítico to tipo Ziegler [10], sem suporte para o catalisador, e caracterizar a estabilidade térmica e flamabilidade dos nanocompósitos obtidos.

\section{MATERIAIS E MÉTODOS}

\section{I Materiais}

Como carga mineral na produção dos nanocompósitos foi usado: i) argila bentonita (fração verde maçia) oriunda do depósito da Companhia Brasileira Bentonita (CBB) em Vitória da Conquista (Bahia); ii) grafita em pó comercial da Windauto Ind. e Com. Ltda; e iii) talco comercial da Johnson \& Johnson.

Para dar um caráter organofílico à argila bentonítica desagregada, esta foi tratada com brometo de hexadeciltrimetil amônio (Cetremide) da Vetec, conforme descrito por Valanzuela-Diaz [I I]. A grafita foi usada diretamente como comercializada e o talco foi tratado termicamente a $450^{\circ} \mathrm{C}$ para remoção de aromatizantes e outros produtos orgânicos.

Para as reações de polimerização os reagentes usados foram: etileno $(99,99 \%$, White Martins SA), nitrogênio (99,99\%, White Martins SA), hexano (99,99\%, Merck), solução de $\mathrm{TiCl}_{4}(\mathrm{I} \mathrm{mol} / \mathrm{L}$ em cloreto de metileno em hexano, Sigma-Aldrich), solução de trietilalumínio (TEAL) (93\% Sigma-Aldrich), sódio metálico (99,99\%, Merck), benzofenona ( $99,99 \%$, Merck) e etanol (99,99\%, Merck).

\subsection{Produção de Polímeros e Nanocompósitos}

As reações de polimerização de etileno foram realizadas em um reator de $250 \mathrm{~mL}$ da Parr Instruments (Série 4560) que tem entrada de gás, ajuste de velocidade de agitação e controle de temperatura (Figura I).

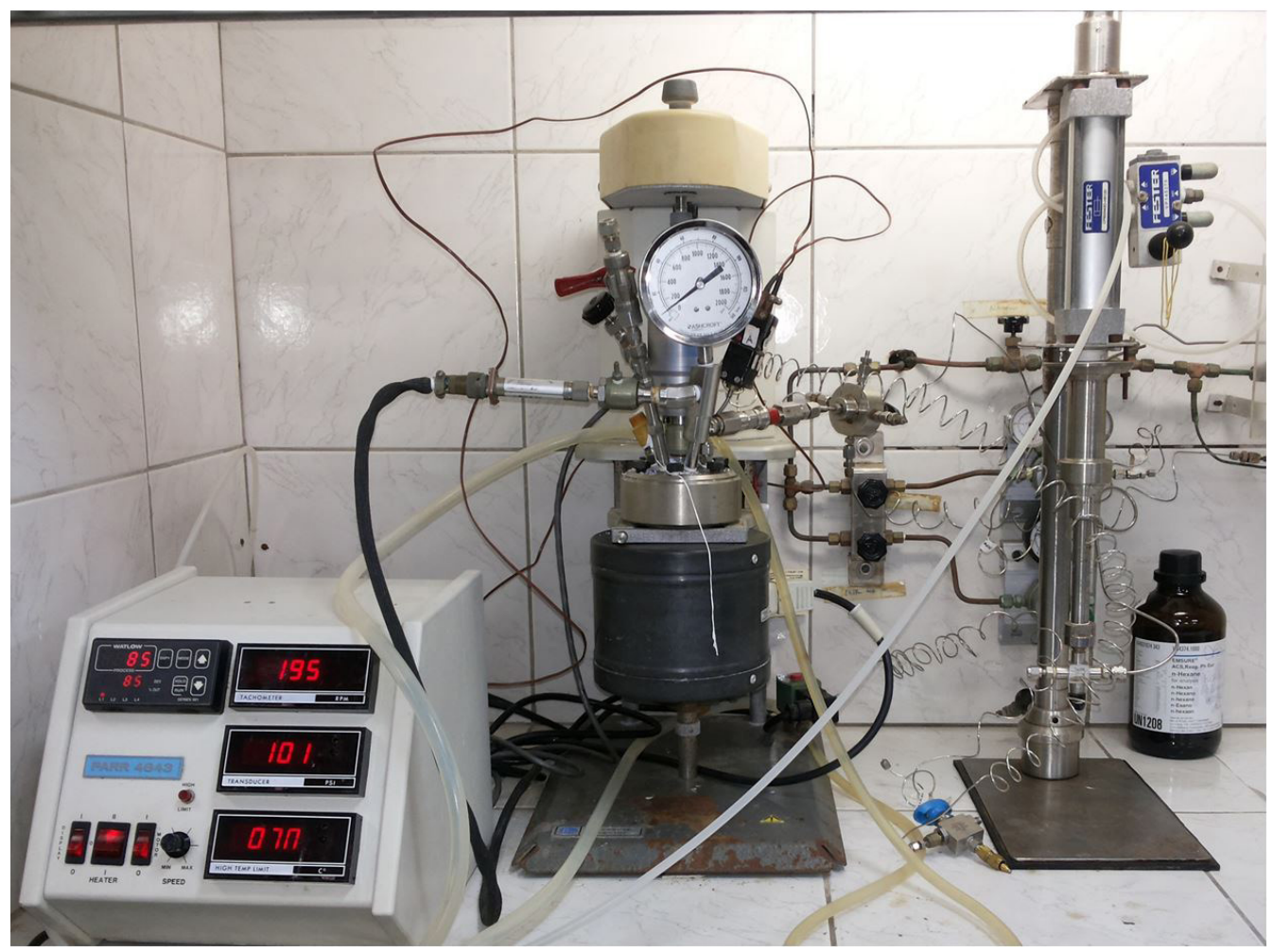

Figura I. Sistema reacional para produção de polietileno e nanocompósitos. 
Inicialmente o reator foi purgado com nitrogênio, $150 \mathrm{~mL}$ de hexano seco foi adicionado. Na produção de nanocompósitos a carga mineral seca foi introduzida nesta etapa. Em seguida, uma solução de $\mathrm{TiCl}_{4}$ em hexano ( 0,3 ou $0,8 \mathrm{~mL}$ de $\mathrm{TiCl}_{4}$ em $10 \mathrm{~mL}$ dehexano) euma solução de TEAL em hexano $(0,9, \mathrm{I}, 8$ ou $2,6 \mathrm{~mL}$ de TEAL em $10 \mathrm{~mL}$ de hexano) foram adicionadas para atuarem como catalisador. A injeção de etileno neste sistema iniciou a polimerização que foi descontinuada após 60 ou 90 minutos por despressurização do reator e adição de $10 \mathrm{~mL}$ de etanol. As polimerizações foram realizadas em temperatura entre 77 e $85^{\circ} \mathrm{C}$ com agitação constante de 190 rpm e pressão entre 2, I e 2,7 bar. Depois de encerrada a reação, o conteúdo do reator foi filtrado a vácuo e lavado usando etanol e depois água destilada para remover o hexano e os resíduos catalíticos. Após esta operação, o polímero foi seco a $80^{\circ} \mathrm{C}$ durante 24 horas. A razão molar ( $\mathrm{Al} / \mathrm{Ti}$ ) utilizada na polimerização foi de 20 ou 29. As condições experimentais para a síntese dos nanocompósitos estão sumarizadas na Tabela I.

\subsection{Caracterização da Carga Mineral e dos Nanocompósitos}

As cargas minerais dos nanocompósitos (argila, grafita e talco) foram caracterizadas previamente aos testes de polimerização. A análise química dos maiores constituintes da argila in natura (fração verde maçia) foi realizada usando fluorescência de raios $X$ com o equipamento S2 Ranger da Bruker. Foram feitos também os testes para caracterização de argilas baseados na extração solução Mehlich I $\left(\mathrm{HCl} \mathrm{0,05mol} / \mathrm{L} \mathrm{e}_{2} \mathrm{SO}_{4} 0,0125 \mathrm{~mol} / \mathrm{L}\right)$ como extrator de $\mathrm{Na}$ e $\mathrm{K}$ trocável e solução de $\mathrm{KCl} I \mathrm{~mol} / \mathrm{L}$ para a extração de $\mathrm{Ca}, \mathrm{Mg}$ e $\mathrm{Al}$ trocáveis. A determinação dos elementos nos extratos e matéria orgânica foram feitas por volumetria ou espectrofotometria. A composição mineralogia das cargas foram avaliadas por difração de raios $X$ utilizando os difratômetros Shimadzu XRD-6000 e Olympus BTX-485, ambos operando com fonte de cobre. A estabilidade térmica da argila organofílica foi avaliada usando análise termogravimétrica (ATG) (com Shimadzu TGA-50) em atmosfera inerte com nitrogênio, para taxas de aquecimento de $5,10,15$ e $20^{\circ} \mathrm{C} / \mathrm{min}$. A argila foi caracterizada por microscopia eletrônica de varredura usando o MEV Hitachi S-3200H.

As temperaturas de fusão dos polímeros nos nanocompósitos foram obtidas por calorimetria diferencial de varredura (DSC) usando o Shimadzu DSC-50, em atmosfera inerte com nitrogênio. A cristalinidade dos polímeros $(\chi c)$ foi estimada a partir da entalpia de fusão das amostras $\left(\Delta \mathrm{H}_{0}\right.$ Amostra estimada como a área sob o pico endotérmico/taxa de aquecimento) utilizando como referência o valor de $\Delta \mathrm{H}_{\mathrm{f}}^{0}$ igual a $293 \mathrm{~J} / \mathrm{g}$ para polietileno de alta massa molar (PEAD) $100 \%$ cristalino, corrigidos para as massas de polímero contidas nos nanocompósitos (Equação I):

$$
\chi_{\mathrm{c}}=\left(\frac{\Delta \mathrm{H}_{\text {Amostra }}^{\mathrm{o}}}{\Delta \mathrm{H}_{\mathrm{f}}^{\mathrm{o}}}\right) \times 100
$$

A estabilidade térmica dos nanocompósitos foi avaliada com ATG em atmosfera inerte usando o DTG-60H da Shimadzu e taxas de aquecimento de $5,10,15$ e $20^{\circ} \mathrm{C} / \mathrm{min}$. A cinética para degradação térmica da maior parte dos polímeros pode ser descrita como um processo de uma única etapa pela Equação 2:

$$
\frac{\mathrm{d} \alpha}{\mathrm{dt}}=\mathrm{A} \exp \left(\frac{-\mathrm{E}_{\mathrm{a}}}{\mathrm{RT}}\right) \mathrm{f}(\alpha)
$$

onde $\alpha$ é a conversão $\left(\alpha=\left(m_{i}-m_{T}\right) /\left(m_{i}-m_{f}\right) ; m_{T}\right.$ é a massa na temperatura $T ; m_{i}$ é a massa inicial e $m_{f}$ é a massa final); $E_{a}$ é a energia de ativação de Arrhenius; A é o fator pré-exponencial; $\mathrm{R}$ é a constante universal dos gases; $\mathrm{T}$ é a temperatura; e $\mathrm{f}(\alpha)$ é o modelo matemático da reação.

Neste estudo a energia de ativação aparente para a degradação do polímero foi estimada usando ATG para distintas taxas de aquecimento usando Shimadzu TGA-50, sob atmosfera inerte de nitrogênio. A energia de ativação foi estimada usando o método de Ozawa-Flynn-Wall para converses entre 20 e $90 \%$, através do gráfico de Arrhenius da

\begin{tabular}{|c|c|c|c|c|c|c|c|}
\hline TESTE & SóLIDO & $\begin{array}{c}\text { TEMPERATURA } \\
\left({ }^{\circ} \mathrm{C}\right)\end{array}$ & $\begin{array}{c}\text { TEMPO DE } \\
\text { REAÇÃO } \\
\text { (min) }\end{array}$ & $\begin{array}{c}\text { AGITAÇÃO } \\
\text { (RPM) }\end{array}$ & $\begin{array}{l}\text { VOLUME DE } \\
\text { TiCL }_{4} \\
(\mathrm{~mL})\end{array}$ & $\begin{array}{l}\text { VOLUME DE } \\
\text { TEAL } \\
(\mathrm{mL})\end{array}$ & $\begin{array}{c}\text { MASSA DA } \\
\text { CARGA } \\
\text { (g) }\end{array}$ \\
\hline $\mathrm{BI}$ & Argila & 85 & 90 & 190 & 0,8 & 2,6 & 0,03 \\
\hline B2 & Argila & 85 & 90 & 190 & 0,8 & 2,6 & 0,05 \\
\hline B3 & Argila & 85 & 90 & 190 & 0,8 & 2,6 & 0,14 \\
\hline B4 & Argila & 85 & 90 & 190 & 0,8 & 2,6 & 0,2 \\
\hline B5 & Argila & 85 & 90 & 190 & 0,8 & 2,6 & 0,3 \\
\hline B6 & Argila & 85 & 90 & 190 & 0,4 & $\mathrm{I}, 3$ & $0, I$ \\
\hline B7 & Argila & 85 & 90 & 190 & 0,4 & 1,3 & 0,2 \\
\hline GI & Grafita & 85 & 90 & 190 & 0,8 & 2,6 & 0,3 \\
\hline $\mathrm{G} 2$ & Grafita & 85 & 90 & 190 & 0,8 & 2,6 & 0,3 \\
\hline TI & Talco & 85 & 90 & 190 & 0,8 & 2,6 & 0,3 \\
\hline
\end{tabular}

Tabela I. Condições experimentais usadas nas reações de polimerização 
taxa de aquecimento $(\beta)$ e da temperatura para conversões definidas (Ti). A inclinação do gráfico ln $\beta$ versus I/Ti é $\mathrm{E}_{\mathrm{a}} / \mathrm{R}$ [I2].

A cristalinidade dos polímeros nos nanocompósitos foram qualitativamente avaliadas usando DRX como fonte de cobre, pois sabe-se que a fração cristalina do polietileno gera um padrão de difração caracterizado por dois picos para $2 \theta$ igual a 22 e 24 graus, correspondentes aos planos ( I 10) e (200) da estrutura cristalina ortorrômbica e a componente amorfa gera um halo para $2 \theta$ entre 20 e 25 graus. Estas características podem ser vistas mais claramente no gráfico $s^{2} \mathrm{l}(\mathrm{s})$ versus $\mathrm{s}$, onde $\mathrm{s}=2 \sin \theta / \lambda$, I é a intensidade do sinal (em contagens por segundo) e $\lambda$ é o comprimento de onda da fonte de raios $X$ [13].

Para avaliação da flamabilidade das amostras de polímero e nanocompósitos foi realizado um teste simples, não padronizado, devido à reduzida massa de material disponível, mas que fornece indícios sobre esta propriedade [I4, I5]. Corpos de prova circulares foram confeccionados usando um molde de $13 \mathrm{~mm}$ de diâmetro, 0,02 gramas de cada amostra e uma pressão de $2 \mathrm{kN}$. Estas pastilhas foram colocadas em contato, na horizontal, com a chama de um bico de Bunsen por 3 segundos e o tempo de queima, assim como o aspecto do resíduo, foi avaliado.

\section{RESULTADOS E DISCUSSÃO}

\section{I Caracterização da Carga Mineral}

A análise química dos maiores constituintes da argila bentonitica in natura é apresentada na Tabela 2 . Nota-se predominância de silício, alumínio, ferro e magnésio. As análises dos extratos da argila (Tabela 3) mostram que o cátion trocável predominante é $\mathrm{Mg}^{2+}$ seguido de $\mathrm{Ca}^{2+}$. A capacidade de troca de cátions (CTC) da argila é muito alto, compatível com a presença de montmorilonita.

A Figura 2 mostra os padrões de difração das cargas minerais usadas neste estudo. $O$ padrão da argila mostra a presença dos picos característicos da montmorilonita $\left((\mathrm{Na}, \mathrm{Ca})_{0.3}(\mathrm{Al}, \mathrm{Mg})_{2} \mathrm{Si}_{4} \mathrm{O}_{10}\left(\mathrm{OH}_{2} \cdot \mathrm{nH}_{2} \mathrm{O}\right)\right)$ e caolinita $\left(\mathrm{Al}_{2} \mathrm{Si}_{2} \mathrm{O}_{5}(\mathrm{OH})_{4}\right)$. Nota-se que o pico característico da argila ocorre em $2 \theta$ igual a 5,6 e corresponde a uma distancia basal de I,58 nm. Os padrões de difração da grafita e do

Tabela 2. Análise química da argila in natura da CBB (fraçao verde maçia)

\begin{tabular}{cc}
\hline Elementos & $\mathbf{( \% )}$ \\
\hline $\mathrm{SiO}_{2}$ & $6 \mathrm{I}, 4$ \\
$\mathrm{Al}_{2} \mathrm{O}_{3}$ & $2 \mathrm{I}, 9$ \\
$\mathrm{Fe}_{2} \mathrm{O}_{3}$ & 10,3 \\
$\mathrm{MgO}$ & 5,30 \\
$\mathrm{TiO}_{2}$ & 0,494 \\
$\mathrm{CaO}$ & 0,188 \\
$\mathrm{Cr}_{2} \mathrm{O}_{3}$ & 0,162 \\
$\mathrm{Total}$ & 99,7 \\
\hline
\end{tabular}

talco comerciais indicam a presença quase que exclusiva dos minerais respectivo, grafita e talco $\left(\mathrm{Mg}_{3} \mathrm{Si}_{4} \mathrm{O}_{10}(\mathrm{OH})_{2}\right)$.

A Figura 3 mostra as imagens em elétrons secundários da argila in natura. Nota-se a estrutura porosa e composta por camadas, característica de presença de montmorilonita.

A análise termogravimétrica para a argila organofílica, tratada com Cetremide, em atmosfera inerte (Figura 4) mostra que a argila apresenta perda de massa em três etapas: i) Entre 20 e $90^{\circ} \mathrm{C}$, que corresponde à perda de água adsorvida que volatiliza em baixas temperaturas; ii) entre 200 e $350^{\circ} \mathrm{C}$ perda da água estrutural e volatilização de compostos orgânicos; e iii) na região acima de $350^{\circ} \mathrm{C}$ que corresponde à deshidroxilação da montmorilonita.

\subsection{Caracterização dos Nanocompósitos}

As massa de nanocompósitos produzidas nos testes de polimerização estão sumarizadas na Tabela 4, nota-se que este valor variou I,23 a 10,74 g. A fração de massa de sólido residual das amostras de nanocompósitos após

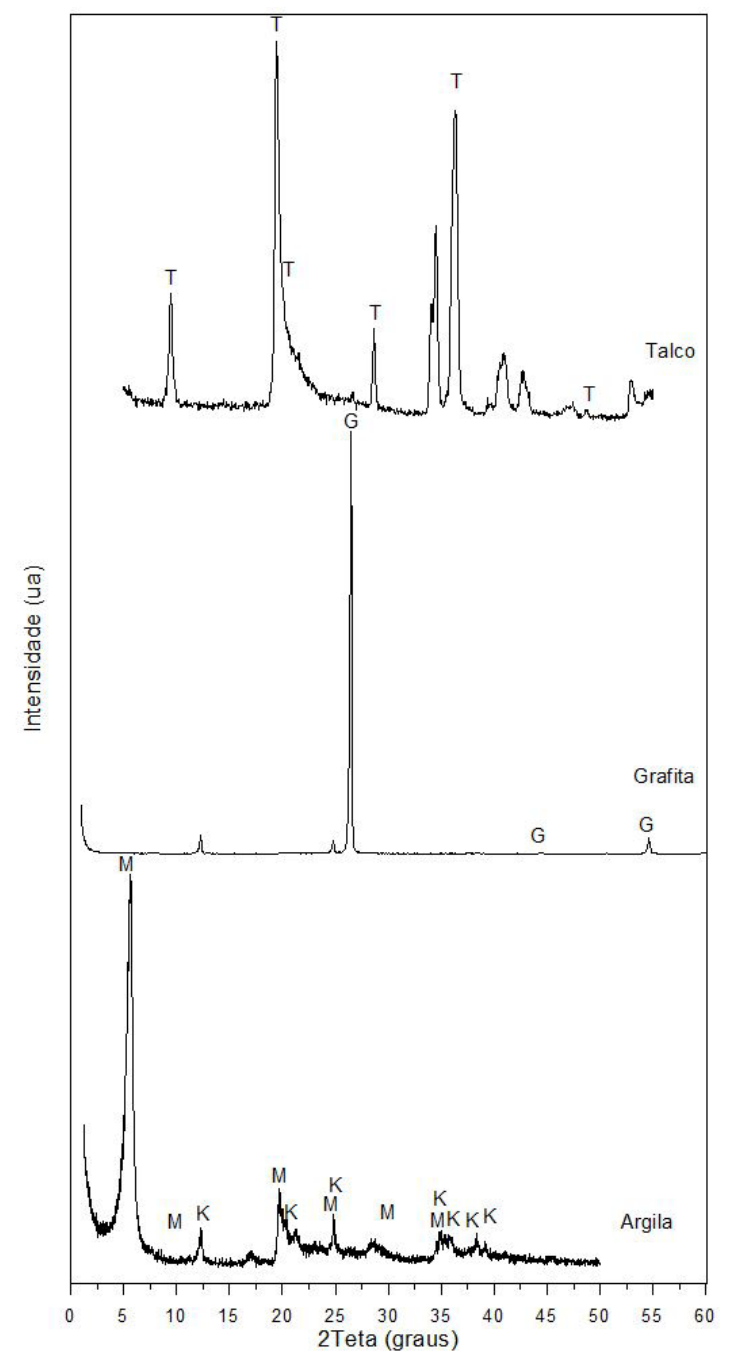

Figura 2. Padrões de difração das cargas inorgânicas (argila, grafita e talco). Minerais identificados: M: montmorilonita; K: caolinita; G: grafita; e T: talco. 

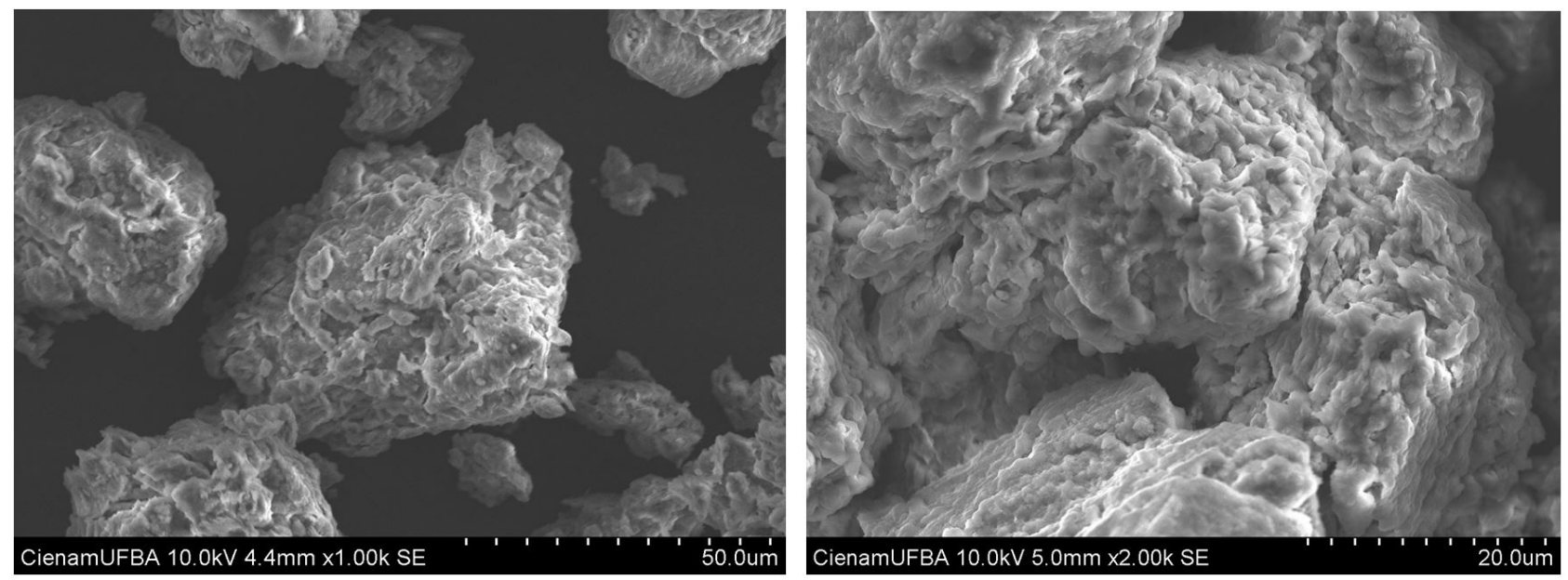

Figura 3. Imagens em elétrons secundários da argila in natura (fração verde maçia da CBB).

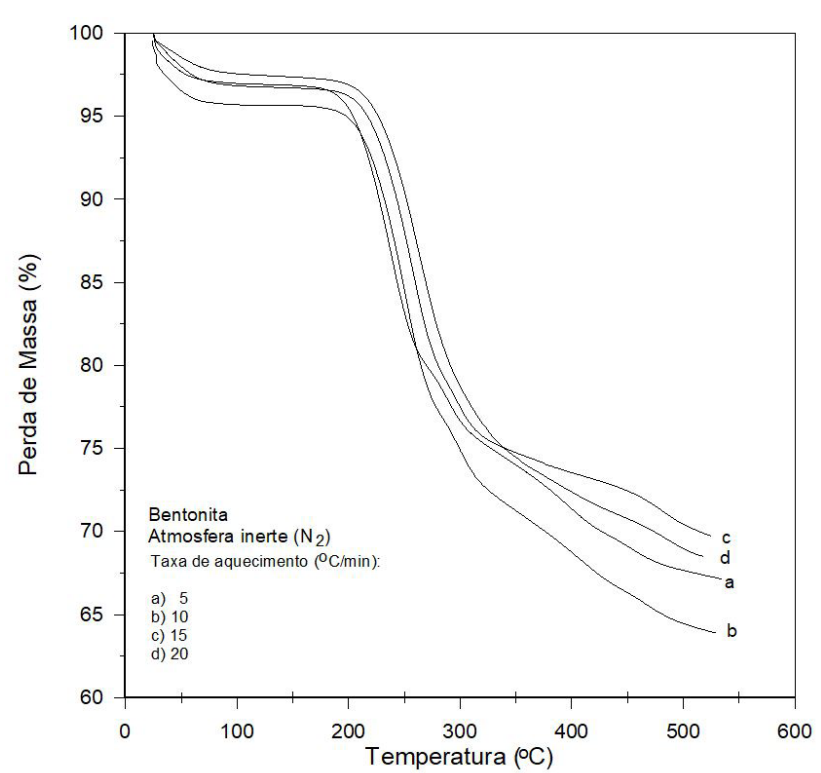

(a)

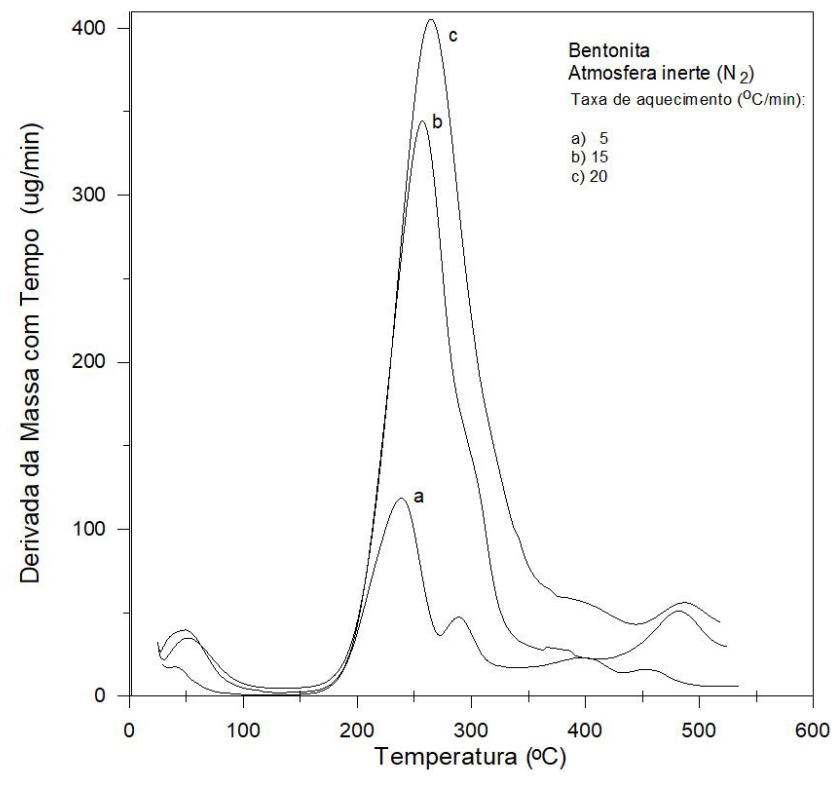

(b)

Figura 4. Degradação térmica da argila organofílica: (a) Análise termogravimétrica (ATG); (b) Derivada da massa com a temperatura (DTG).

Tabela 3. Caracterização da argila da Companhia Brasileira de Bentonita, usada neste estudo após tratamento

\begin{tabular}{|c|c|c|c|}
\hline PARÂMETROS & & SULTAD & \\
\hline Acidez ativa & $\mathrm{pH}$ & 4,8 & (em água) \\
\hline \multirow[t]{4}{*}{ Bases trocáveis: } & $\mathrm{K}$ & 0,08 & $\left(\mathrm{cmol} / \mathrm{dm}^{3}\right)$ \\
\hline & $\mathrm{Ca}$ & 8,00 & $\left(\mathrm{cmol} / \mathrm{dm}^{3}\right)$ \\
\hline & $\mathrm{Mg}$ & 30,50 & $\left(\mathrm{cmol}_{\mathrm{c}} / \mathrm{dm}^{3}\right)$ \\
\hline & $\mathrm{Na}$ & 1,22 & $\left(\mathrm{cmol}_{\mathrm{c}} / \mathrm{dm}^{3}\right)$ \\
\hline Acidez trocável ou alumínio trocável & $\mathrm{Al}$ & 19,6 & $\left(\mathrm{cmol}_{\mathrm{c}} / \mathrm{dm}^{3}\right)$ \\
\hline Acidez total ou potencial & $\mathrm{H}+\mathrm{Al}$ & 20,02 & $\left(\mathrm{cmol}_{\mathrm{c}} / \mathrm{dm}^{3}\right)$ \\
\hline Capacidade de troca de cátions (CTC) & $\mathrm{Ca}^{2+}+\mathrm{Mg}^{2+}+\mathrm{K}^{+}+\mathrm{Na}^{+}+\mathrm{H}+\mathrm{Al}$ & 59,82 & $\left(\mathrm{cmol} / \mathrm{dm}^{3}\right)$ \\
\hline Saturação por bases $(V)$ & & 67 & $(\%)$ \\
\hline Matéria orgânica & & 6,73 & $(g / k g)$ \\
\hline
\end{tabular}


Tabela 4. Características dos nanocompósitos produzidos por polimerização in situ

\begin{tabular}{|c|c|c|c|c|c|}
\hline TESTE & MASSA PRODUZIDA (g) & $\begin{array}{c}\text { SÓLIDOS APÓS } \\
\text { QUEIMA A } 500{ }^{\circ} \mathrm{C}(\%)\end{array}$ & $\begin{array}{l}\text { TEMPERATURA } \\
\text { DE FUSÃO }\left({ }^{\circ} \mathrm{C}\right)\end{array}$ & $\begin{array}{c}\text { ENTALPIA DE } \\
\text { FUSÃO, } \Delta H_{M}(\mathrm{~J} / \mathrm{g})\end{array}$ & $\begin{array}{c}\text { CRISTALINIDADE, } \\
(\%)\end{array}$ \\
\hline $\mathrm{BI}$ & 1,68 & 4,6 & 146,2 & - & - \\
\hline B2 & 1,96 & 5,4 & 133,7 & - & - \\
\hline B3 & 2,40 & 16,4 & 134,9 & - & - \\
\hline B4 & 2,46 & 9,1 & 133,8 & - & - \\
\hline B5 & 5,67 & 6,8 & 137,4 & 128,6 & 47 \\
\hline B6 & 2,62 & 4,0 & 133,6 & 158,5 & 56 \\
\hline B7 & $\mathrm{I}, 23$ & - & 133,4 & 130,6 & - \\
\hline GI & 3,82 & 8,0 & $|40|$, & 153,2 & 57 \\
\hline $\mathrm{G} 2$ & 10,74 & $\mathrm{II}, \mathrm{I}$ & 140,1 & 149,5 & 57 \\
\hline TI & 5,29 & 5,6 & 133,3 & 160,6 & 58 \\
\hline
\end{tabular}

a degradação térmica a $500{ }^{\circ} \mathrm{C}$ em atmosfera oxidante indicam um valor considerável de sólidos. Foi observado que as amostras de nanocompósitos $\mathrm{BI}$ a B6 têm cerca de 4,0 a $16,4 \%$ de resíduos sólidos, enquanto as amostras de nanocompósitos GI, G2 e TI têm cerca de 8, I I, I e 5,6\% de resíduos sólidos. Estes sólidos são compostos pela carga mineral e resíduo de alumínio e titânio usados como catalisador.

As curvas de DSC para atmosfera inerte são mostradas nas Figuras 5a e 5b. Nota-se a presença de eventos endotérmicos em baixa temperatura (fusão) e em alta temperatura (degradação térmica dos polímeros). A temperatura de fusão do polímero nos nanocompósitos apresentou uma variação de 133 a $146^{\circ} \mathrm{C}$, confirmando a presença de polietileno de alta massa molar em todos os casos e o grau de cristalização do polímero nos nanocompósitos esta em torno de $57 \%$ (Tabela 4).

Os padrões de raios $X$ da argila in natura, organofilizada e no nanocompósito polietileno/argila, na região de baixo ângulo (Figura 6) mostram que houve um deslocamento das camadas da argila (in natura: $2 \theta=5.6$ graus e $d=1.58 \mathrm{~nm}$, tratada: $2 \theta=4.7$ graus e $d=1.88 \mathrm{~nm}$, no nanocompósito: $2 \theta=2$ teta $=4.5$ graus e $d=1.96 \mathrm{~nm}$ ) indicando intercalação no processo de organofilização e polimerização.

A Figura 7 mostra as difrações de raios- $X$ dos nanocompósitos polietileno-argila e compara com o padrão de difração obtido para polietileno de alta massa molar puro, também produzido em laboratório [13]. Observa-se para todos os casos que os dois picos característicos da cristalização ortorrômbica estão presentes. Nota-se também o halo característico da presença de estrutura amorfa e que a proporção destas regiões com os picos são análogas para todas as amostras.

A Figura 8 mostra a análise termogravimétrica para o polietileno obtido em laboratório e o nanocompósito com argila produzido por polimerização in situ, em atmosfera inerte. Pode-se notar que ambas amostras degradam em uma única etapa que inicia próximo de $350^{\circ} \mathrm{C}$ e termina próximo de $500{ }^{\circ} \mathrm{C}$. Nota-se, entretanto, que a amostra com carga mineral (nanocompósito) apresenta um pequeno

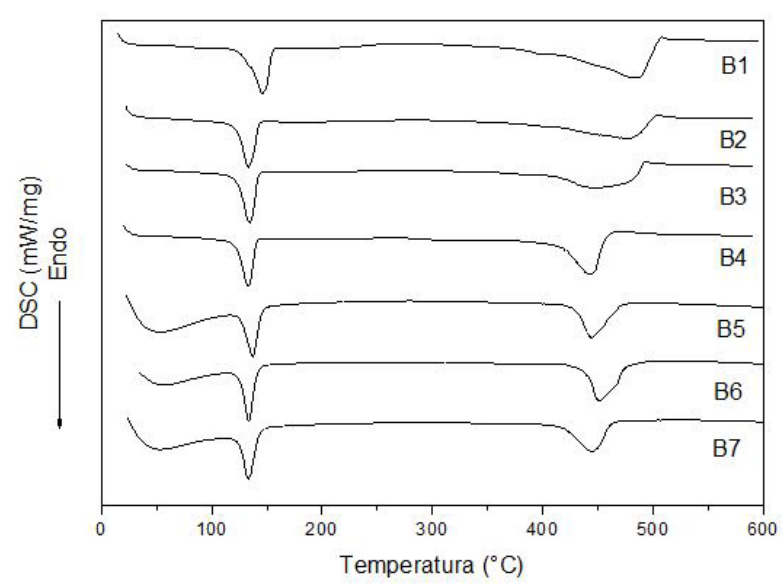

(a)

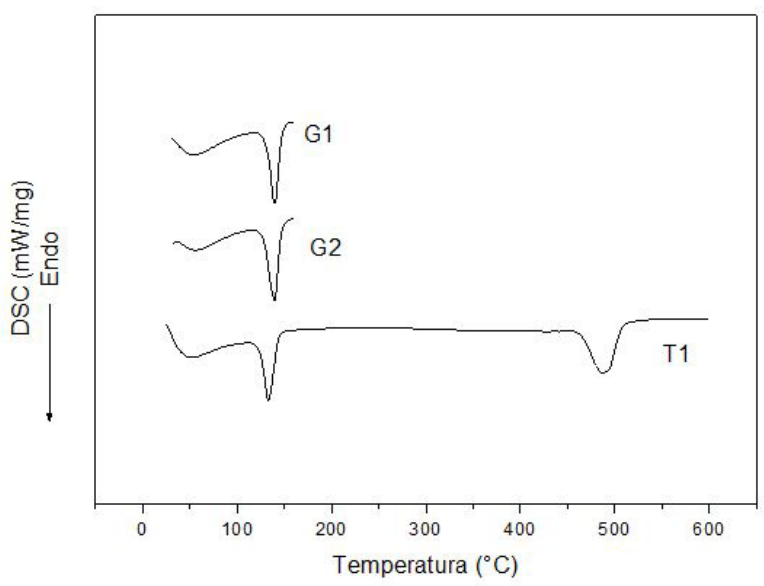

(b)

Figura 5. DSC dos nanocompósitos: (a) com argila (Bi); (b) Com grafita (GI e G2) e talco (TI).

deslocamento para direita indicando uma maior estabilidade térmica.

As Figuras 9a a 9d mostram as curves de análise termogravimétrica para degradação dos 


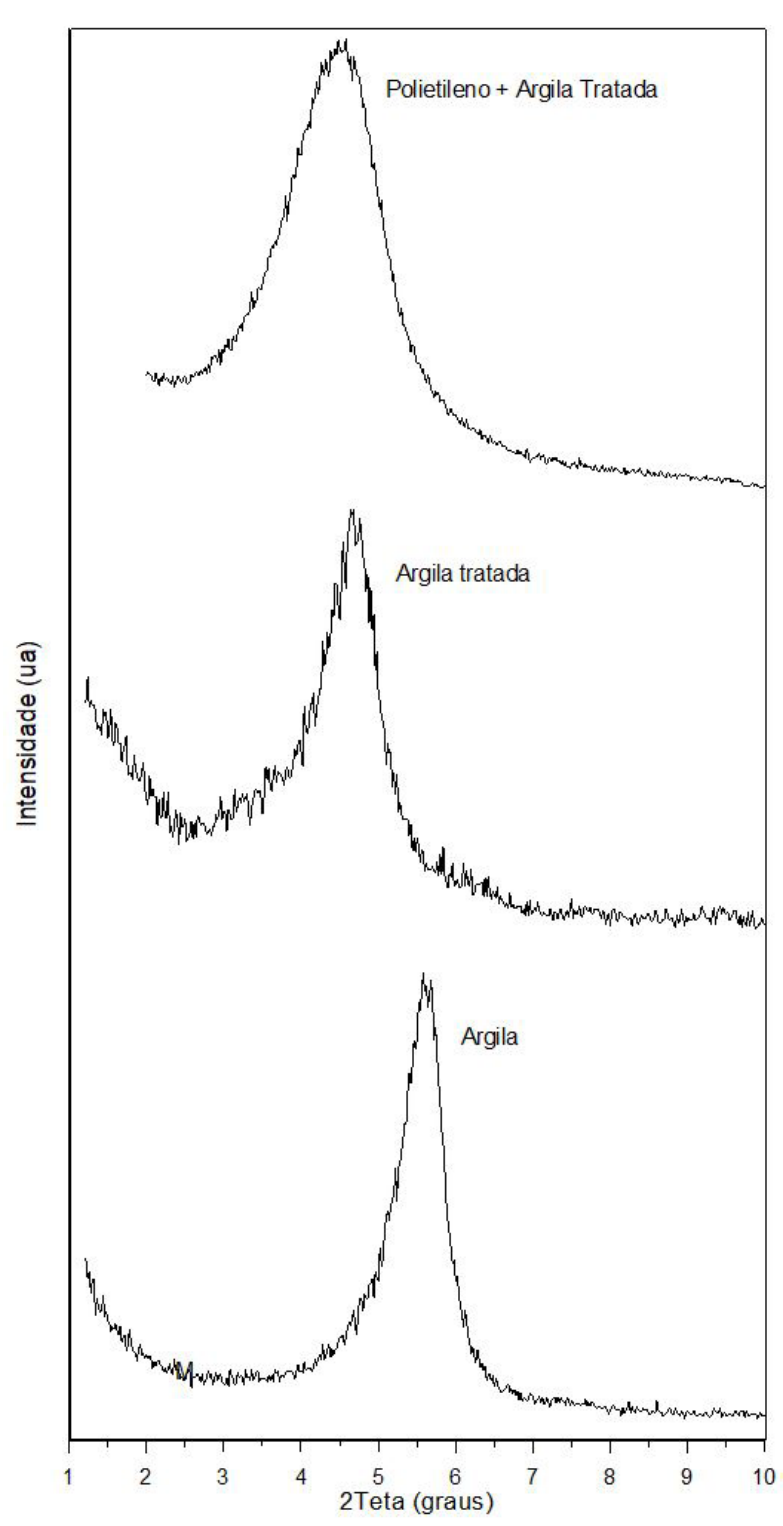

Figura 6. Padrões de difração para argila in natura, argila tratada com Cetremide e argila após a polimerização.

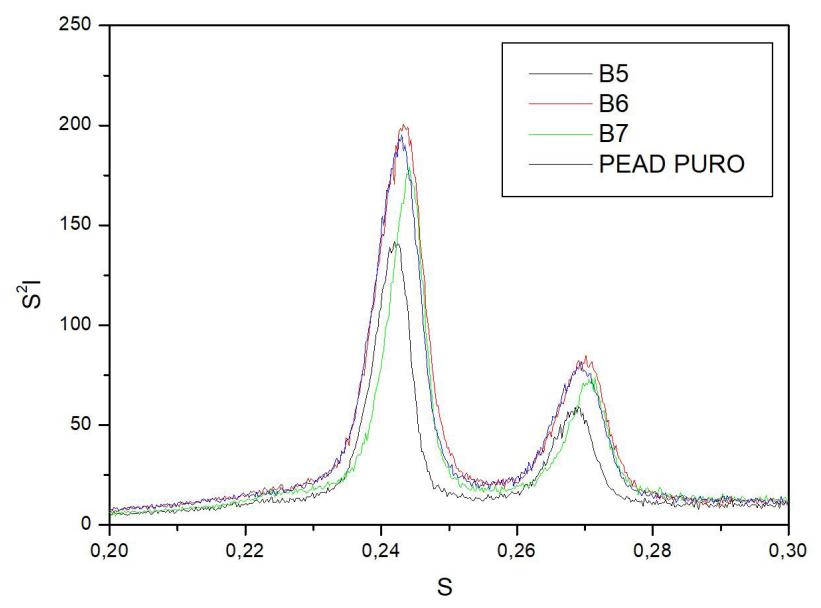

Figura 7. Curvas de $\mathrm{s}^{2} \mathrm{I}(\mathrm{s})\left(\right.$ em c.p.s. $\left./ \AA^{2}\right)$ versus $\mathrm{s}$, onde $\mathrm{s}=2 \sin \theta / \lambda$ $\left(\right.$ em $\left.\AA^{-1}\right)$, dos nanocompósitos (B5, B6 e B7) e do polímero (PEAD PURO). nanocompósitos em atmosfera inerte para quatro taxas de aquecimento $\left(5,10,15\right.$ e $\left.20^{\circ} \mathrm{C} / \mathrm{min}\right)$. Nota-se que a degradação tem início próximo de $400{ }^{\circ} \mathrm{C}$ mas apresentam comportamentos diferentes para cada carga usada. A energia de ativação aparente para a degradação térmica dos nanocompósitos é mostrada na Figura 10 em função da conversão. Nota-se um valor próximo de $200 \mathrm{~kJ} / \mathrm{mol}$ para os nanocompósitos com argila e cerca de $300 \mathrm{~kJ} / \mathrm{mol}$ para os nanocompósitos com grafita, Estes valores são mais altos que os valores encontrados na literatura para polietileno de alta massa molar, indicando que há uma melhoria na estabilidade térmica do polímero nos nanocompósitos [16].

A Tabela 5 mostra os resultados de tempo de queima para o polietileno puro e para os nanocompósitos. A amostra de polímero puro (AI) queimou totalmente em 5 segundos, ocorrendo o consumo de toda o material. Todas as amostras de nanocompósitos com argila (B2 a B6) queimaram parcialmente (apagaram após certo tempo de queima deixando material remanescente) e o tempo que resistiram foi entre 7 e 20 segundos. A amostra de nanocompósitos com grafita ( $\mathrm{GI}$ ) não queimou (iniciou e parou) e a amostra $\mathrm{G} 2$ queimou por 30 segundos. A amostra de nanocompósitos com talco ( $\mathrm{TI}$ ) queimou por 30 segundos. Os valores obtidos no teste mostraram que o aumento do teor de argila organofílica nos sistemas teve uma tendência em aumentar o tempo de queima e que os nanocompósitos com grafita e talco apresentaram tempos de queima semelhantes e superiores aos tempos de queima dos nanocompósitos com argila organofílica. Essas mudanças na forma como o polímero queima no nanocompósito ocorre devido a formação de uma camada de carga mineral que atua como barreira, isolando os gases e produtos voláteis da degradação $[|4| 5$,$] .$

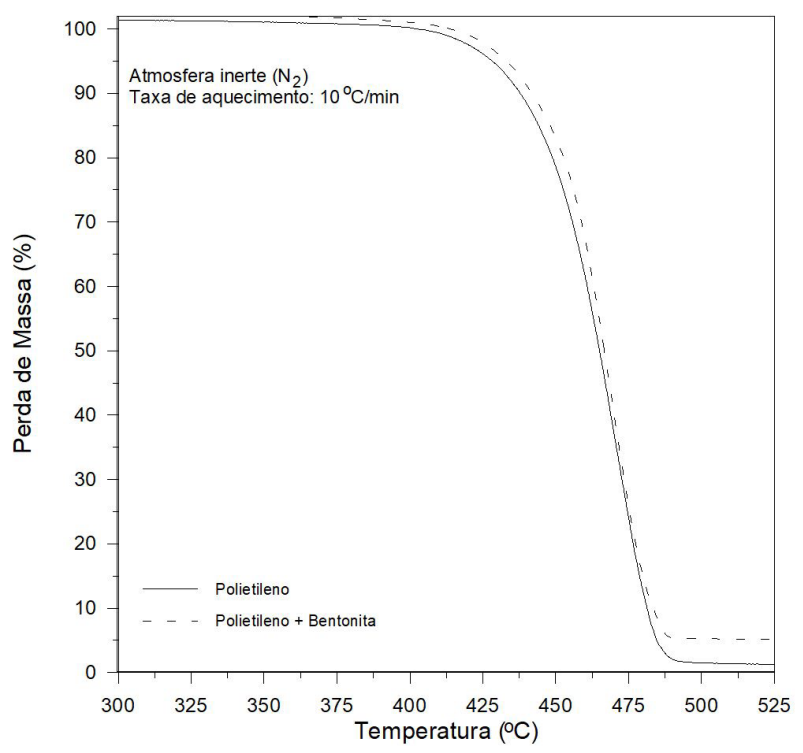

Figura 8. Degradação térmica do polímero puro e no nanocompósito com argila. 


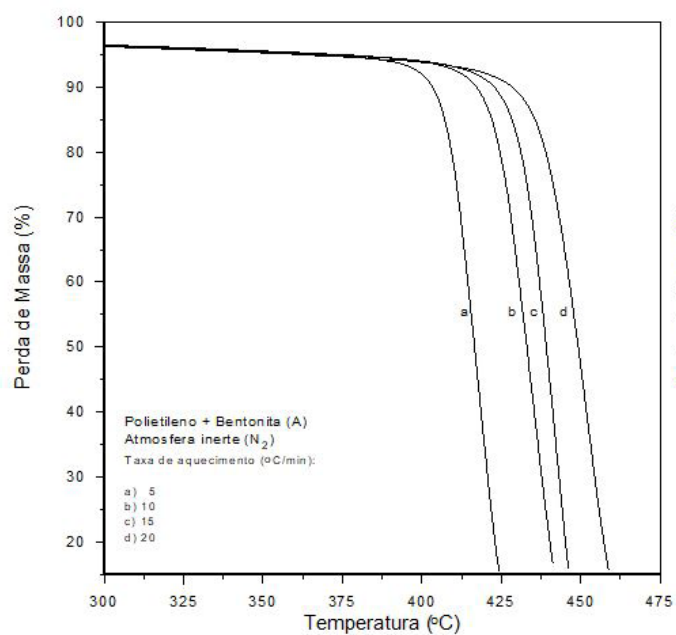

(a)

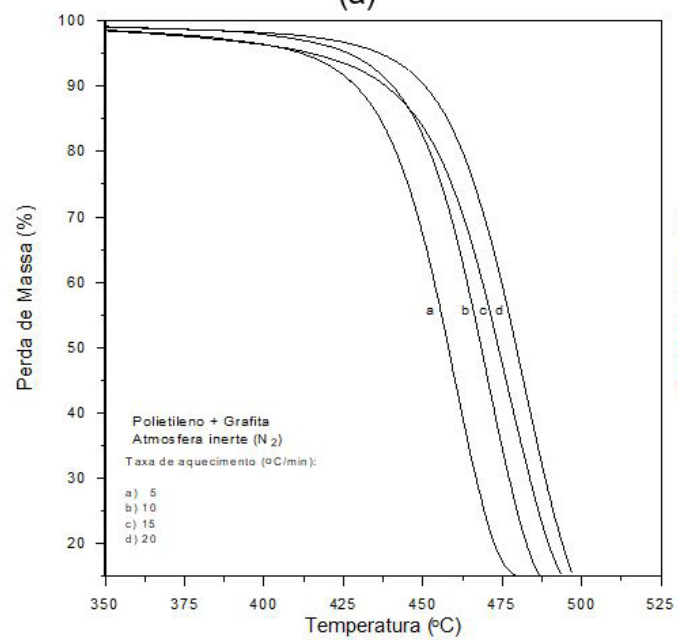

(c)

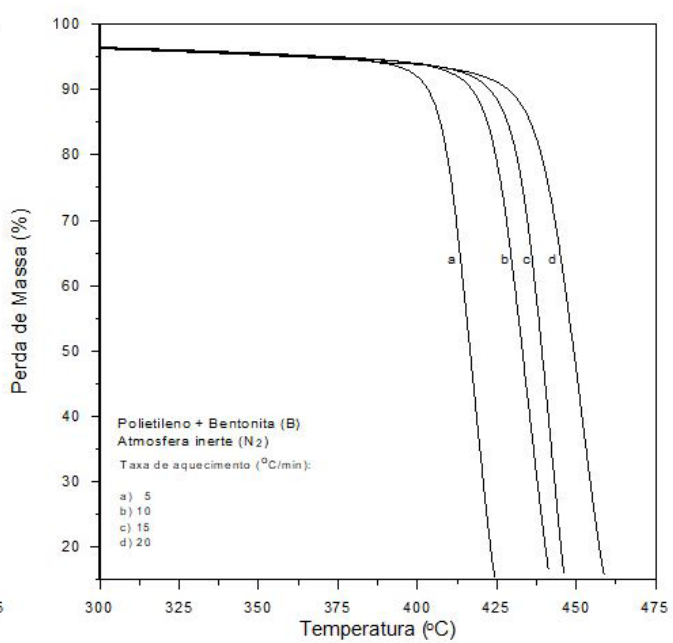

(b)

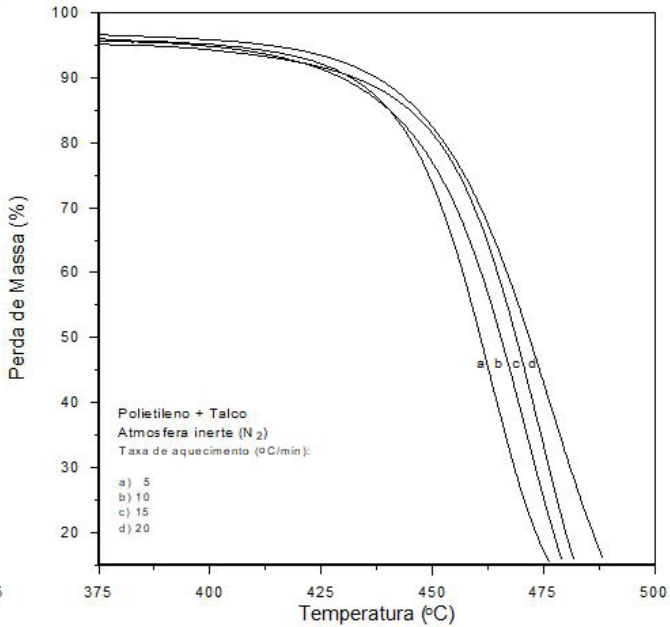

(d)

Figura 9. Cinética de degradação térmica do polímero nos nanocompósitos. (a) polietileno-argila; (b) polietileno-argila; (c) polietileno-grafita; (d) polietileno-talco.

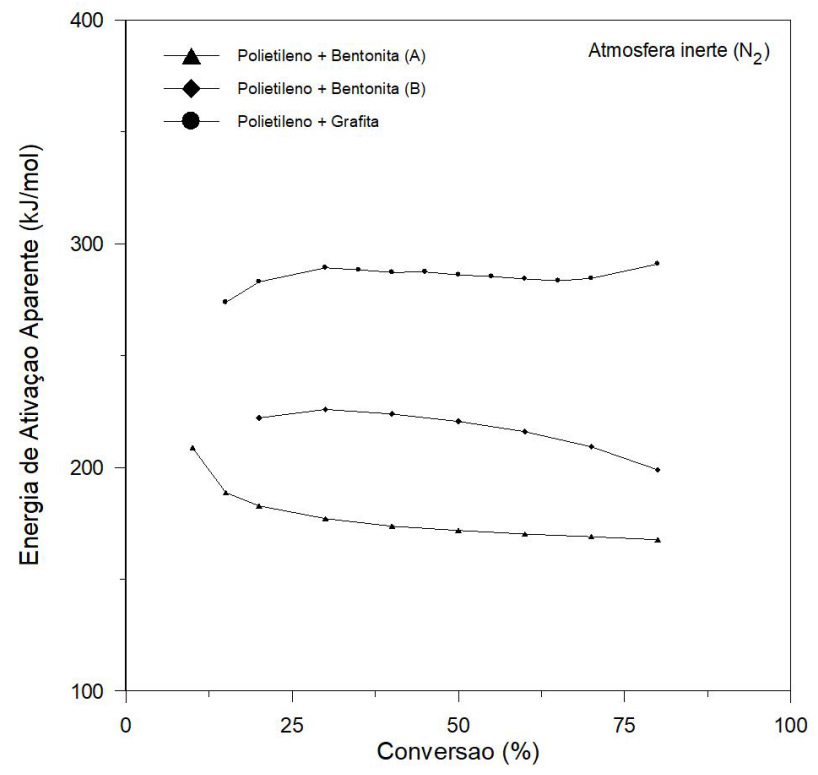

Figura 10. Energia de ativação aparente para degradação térmica do polímero nos nanocompósitos em função da conversão.
Tabela 5. Resultados dos tempos de queima do teste de flamabilidade

\begin{tabular}{ccc}
\hline AMOSTRA & $\begin{array}{c}\text { TEMPO DE } \\
\text { QUEIMA (s) }\end{array}$ & OBSERVAÇÃO \\
\hline AI & 5 & Queima total \\
B2 & 13 & Queima parcial, chama extinguiu \\
B3 & 17 & Queima parcial, chama extinguiu \\
B4 & 7 & Queima parcial, chama extinguiu \\
B5 & 20 & Queima parcial, chama extinguiu \\
B6 & 15 & Queima parcial, chama extinguiu \\
GI & 5 & Queima incipiente, chama extinguiu \\
G2 & 30 & Queima total \\
TI & 30 & Queima total \\
\hline
\end{tabular}

\section{CONCLUSÕES}

Nanocompósitos de polietileno e argila, grafita e talco foram produzidos usando polimerização direta em solventes com um sistema catalítico do tipo Ziegler ( $\mathrm{TEAL} \mathrm{e} \mathrm{TiCl}_{4}$ ). Os nanocompósitos foram caracterizados 
demonstrando que polietileno de alta massa molar e com cristalinidade de cerca de $57 \%$ foram obtidos. A degradação térmica em atmosfera inerte do polietileno puro obtido tem inicio em temperatura acima de $400{ }^{\circ} \mathrm{C}$ enquanto o polietileno nos nanocompósito apresentou um deslocamento para região de altas temperaturas indicando um aumento da estabilidade térmica da matriz polimérica. Os testes de flamabilidade mostraram um efeito de redução ou atenuação da progressão das chamas para os nanocompósitos (tanto com argila quanto com grafita ou talco) devido ao efeito de barreira para gases e compostos voláteis.

\section{AGRADECIMENTOS}

Este estudo foi suportado financeiramente pelo Conselho Nacional de Desenvolvimento Científico e Tecnológico (CNPq, Brasil) projetos no: I57 I96/20 I I-9 e 458098/20 I4-0. Jonei Marques da Costa (IFBA) é agradecido pelo fornecimento das amostras de argila usadas neste estudo e por realizar análises de XRF deste material. Zênis Novais da Rocha (IQ-UFBA) é agradecida por participar na síntese dos polímeros e nanocompósitos. Os autores agradecem também as análises térmicas e de DRX realizadas nos laboratórios: i) Catálise e Polímeros, ii) Energia e Ciência dos Materiais e iii) Pesquisa em Catálise e Materiais da Universidade Federal da Bahia.

\section{REFERÊNCIAS}

I Paul DR, Robeson LM. Polymer nanotechnology: nanocomposites. Polymer. 2008;49:3 I87-3204.

2 Silva BL, Nack FC, Lepienski CM, Coelho LAF, Becker D. Influence of intercalation methods in properties of clay and carbon nanotube and high density polyethylene nanocomposites. Materials Research. 2014;17:1628-1636.

3 Araujo EM, Barbosa R, Rodrigues AWB, Melo TJA, Ito EN. Processing and characterization of polyethylene/Brazilian clay nanocomposites. Materials Science and Engineering A. 2007;445-446: I 4 I- 147.

4 Suarez JCM, Bonello CMC, Eltom AE, Mano EB. Comportamento fisico-químico de uma mistura LDPE/HDPE irradiada: efeito da atmosfera. Tecnologia em Metalurgia e Materiais. 2005;2: 18-23.

5 Pandey JK, Reddy KR, Kumar AP, Singh RP. An overview on the degradability of polymer nanocomposites. Polymer Degradation \& Stability. 2005;88:234-250.

6 Jin YH, Park HJ, Im SS, Kwak S. Polyethylene/clay nanocomposite by in-situ exfoliation of montmorillonite during Ziegler-Natta polymerization of ethylene. Macromolecular Rapid Communications. 2002;23: I 35- I 40.

7 Ramazani A, Tavakolzadeh F, Baniasadi H. In situ polymerization of polyethylene/clay nanocomposites using a novel clay-supported Ziegler-Natta catalyst. Polymer Composites. 2009;30: I388-1393.

8 Nikkhah S, Ramazani A, Baniasadi H, Tavakolzadeh F. Investigation of properties of polyethylene/clay nanocomposites prepared by new in situ Ziegler-Natta catalyst. Materials \& Design. 2009;30:2309-23I 5 .

9 Abedi S, Abdouss M, Haghighi M, Sanjani N. PE/clay nanocomposites produced via in situ polymerization by highly active clay-supported Ziegler-Natta catalyst. Polymer Bulletin. 2013;70:1313-1325.

10 Haag RB, Silva JC. Process for preparing a Ziegler type catalytic system and process for preparing ultra high molecular weight polyethylene. United States patent US 4983693. I99I.

I I Valanzuela-Diaz FR. Preparação a nível de laboratório de algumas argilas organofílicas [tese]. São Paulo: Universidade de São Paulo; 1994.

12 Bruce Prime R, Bair HE, Vyazovkin S, Gallagher PK, Riga A. Thermogravimetric Analysis (TGA). In: Menczel JD, Bruce Prime R, editors. Thermal analysis of polymers: fundamentals and applications. Hoboken: John Wiley \& Sons; 2009.

13 Alexander LE. X-ray diffraction methods in polymer science. New York: Wiley-Interscience; 1970.

14 Tang Y, Lewin M. New aspects of migration and flame retardancy in polymer nanocomposites. Polymer Degradation \& Stability. 2008;93:1986-1995.

15 Hull TR, Stec AA, Nazare S. Fire retardant effects of polymer nanocomposites. Journal of Nanoscience and Nanotechnology. 2008;8:I-9.

16 Khaghanikavkani E, Farid MM. Thermal pyrolysis of polyethylene: kinetic study. Energy Science and Technology. 20II;2:I-I0.

Recebido em: 8 Set. 2019

Aceito em: 7 Fev. 2020 